\title{
Using a scalar parameter to trace dislocation evolution in atomistic modeling
}

\author{
J.B. Yang* ${ }^{* 1,2}$, Z.F.Zhang ${ }^{1}$, Y.N. Osetsky ${ }^{2}$ and R.E. Stoller ${ }^{2}$ \\ ${ }^{1}$ Shenyang National Laboratory for Materials Science, Institute of Metal Research, Chinese Academy of Sciences, \\ 72 Wenhua Road, Shenyang 110016, People's Republic of China \\ ${ }^{2}$ Materials Science and Technology Division, ORNL, One Bethel Valley Road, P.O. Box 2008, MS-6114 Oak Ridge, \\ TN 37831, USA
}

\begin{abstract}
A scalar $\gamma$-parameter is proposed from the Nye tensor. Its maximum value occurs along a dislocation line, either straight or curved, when the coordinate system is purposely chosen. This parameter can be easily obtained from the Nye tensor calculated at each atom in atomistic modeling. Using the $\gamma$-parameter, a fully automated approach is developed to determine core atoms and the Burgers vectors of dislocations simultaneously. The approach is validated by revealing the smallest dislocation loop and by tracing the whole formation process of complicated dislocation networks on the fly.
\end{abstract}

Keywords: dislocation; Nye tensor; atomistic modeling; simulation; plastic deformation

*Corresponding author. Tel: 86-24-23971043; E-mail: jbyang@imr.ac.cn; 


\section{Introduction}

Atomistic modeling such as molecular dynamics simulations has become a powerful tool which allows us to unambiguously examine deformation processes of crystalline materials from an atomistic viewpoint [1]. When the applied loading is approaching the strength of materials during simulations, small loops or segments of dislocations will nucleate and then gradually grow up [2-5]. This evolution process of dislocations usually dominates the deformation behaviors of materials. Therefore, it is desirable to develop dislocation visualization methods to reveal the underlying mechanisms of deformation in atomistic modeling.

Dislocations can be visualized by excess energy analysis [6], common neighbor analysis [7], and other filtering tools [8] because they cause variations of atomic energy and positions. However, these filtering tools provide no information about the Burgers vectors to characterize dislocations. There are two types of methods to obtain the Burgers vectors of dislocations. One is through the Burgers circuit mapping, and various ways of construction and mapping of the Burgers circuit have been proposed [9-13]. The other is by numerical integration of the Nye tensor over an area perpendicular to the dislocation line [14-17]. This integration is relatively time-consuming since it needs an interpolation method to get the accurate shape of spatial distribution of the Nye tensor [14, 17]. It is apparent that the integration could become problematic for closely spaced dislocations because of the overlap of their Nye tensor field. On the other hand, except the case with straight dislocations [18], the Nye tensor cannot be used to detect independently core atoms of curved dislocations because the value of its nine elements could change dramatically with the dislocation line direction. This can be seen from the work of Begau et al [17], in which a filtering tool rather than the Nye tensor was utilized to detect core atoms of curved dislocations.

It is the goal of this paper to develop a simple approach on the basis of the Nye tensor calculation only, which is capable of detecting atoms in dislocation cores without aid of any filtering tool, and at the same time, determining their Burgers vectors by the property of a scalar $\gamma$-parameter we proposed rather than the integration of the Nye tensor mentioned above. In this work, a $\gamma$-parameter is first proposed on the basis of the Nye tensor. Then, using this scalar parameter, a simple approach is developed and implemented into atomistic modeling. Several 
examples are selected to demonstrate the properties of the $\gamma$-parameter and the ability of our approach. Finally, advantages and limitations of our approach are discussed.

\section{Theoretical derivations}

A dislocation is characterized mainly by its Burgers vector that can be described by the Nye tensor [19, 20]. Suppose that a single dislocation with a Burgers vector $\boldsymbol{b}$ (henceforth called dislocation $\boldsymbol{b}$ ) exists in a continuum media. Its line could be straight or curved, denoted by " $L$ ". Its Burgers vector $\boldsymbol{b}$ is defined as

$$
\boldsymbol{b}=-\oint_{C} \boldsymbol{G} d \boldsymbol{y}
$$

where $C$ is a closed circuit around the dislocation line $L, G$ is the lattice distortion tensor, the same as that defined in ref. [14], and $d \boldsymbol{y}$ is an infinitesimal step along the Burgers circuit $C$. When the Stokes' theorem is applied to Eq. (1), we have

$$
\boldsymbol{b}=\int_{A} \operatorname{and} d s
$$

Here $A, d s$, and $\boldsymbol{n}$ are the area bounded by the circuit $C$, an area element and the unit normal vector of the area element, respectively. $\alpha$ is the well-known Nye tensor, the tensor of dislocation density. The nine components of $\alpha$ can be calculated according to

$$
\alpha_{\mathrm{ij}}=-\varepsilon_{\mathrm{jk} l} \partial \mathrm{g}_{\mathrm{il}} / \partial x_{\mathrm{k}}
$$

where $i, j, k$ and $l=1-3$, the permutation tensor $\varepsilon_{\mathrm{jkl}}=(j-k)(k-l)(l-j) / 2$, and the Einstein summation convention is employed. In Eq. (3), $\alpha_{\mathrm{ij}}$ and $g_{\mathrm{il}}$ are the component of $\boldsymbol{\alpha}$ and $\mathbf{G}$, respectively. Eqs. (1)-(3), equivalent to Eqs. (9)-(11) in ref. [14], are briefly outlined here for completeness.

The Nye tensor near the line $L$ of the dislocation $\boldsymbol{b}$ is a function of the line direction and the Burgers vector [19]. Let $P$ be a point of intersection of the dislocation line $L$ and the area $A$, and $l$ be a unit direction vector of the line $L$ at $P$. Following Kröner's Eq. (32) in ref. [21], the Nye tensor at the point $P$ can be written as

$$
\boldsymbol{\alpha}=\boldsymbol{b} \boldsymbol{l}^{T} \delta(\rho)
$$

Here the superscript " $T$ " represents the transpose of a vector. $\delta(\rho)$ is a delta function which is infinite along the dislocation line and zero otherwise, and $\rho$ is the distance to the point P. 
Dislocation cores are assumed to have a negligible width in Eq. (4). This is an idealized situation because dislocation cores are more or less dispersed [22]. Eq. (4) implies that

$$
\boldsymbol{\alpha} \propto \boldsymbol{b l}^{T}
$$

In order to employ $\alpha$ to visualize curved dislocations, it is necessary to propose a new parameter independent to the line direction $\boldsymbol{l}$. For this purpose, a matrix $\boldsymbol{Y}$ is built by the equation:

$$
\boldsymbol{Y}=\boldsymbol{\alpha} \boldsymbol{\alpha}^{T}\left(\propto \boldsymbol{b} \boldsymbol{b}^{T}\right)
$$

The term in the parenthesis is derived from Eq. (5). It is seen from Eq. (6) that all the elements in $\boldsymbol{Y}$, including the $\gamma$-parameter defined later, depend only on the Burger vector, but not a function of the dislocation line direction $\boldsymbol{l}$. Furthermore, the matrix $\boldsymbol{b} \boldsymbol{b}^{\mathrm{T}}$ in Eq. (6) will contain only one nonzero element if $\boldsymbol{b}$ becomes parallel to a coordinate axis. Then, the corresponding nonzero element in $\boldsymbol{Y}$ (the $\gamma$-parameter) is obtained to reveal curved dislocations. This can be achieved by coordinate system transformation, as presented in the following.

Two types of orthogonal coordinate systems are utilized throughout this work. One is the coordinate system (henceforth represented by $\left\{\boldsymbol{x}_{i}\right\}$ ) of the simulation box with three axes that are denoted by the unit vectors $\boldsymbol{x}_{1}, \boldsymbol{x}_{2}$, and $\boldsymbol{x}_{3}$, and the other is established for each type of Burgers vectors. Assume that the Burger vector $\boldsymbol{b}$ has $n$ known values, e.g. they are usually $<111>/ 2$ and $<001>$ translation vectors in a body centered cubic (bcc) crystal, that is, $\boldsymbol{b}=\boldsymbol{b}_{k}(k=1 \cdots n)$. Each $\boldsymbol{b}_{\mathrm{k}}$ has a coordinate system $\left\{{ }^{k} \boldsymbol{x}_{i}\right\}$ with the condition that ${ }^{k} \boldsymbol{x}_{3}$ should be chosen to be parallel to $\boldsymbol{b}_{k}$, as sketched in Fig. 1. In order to distinguish them from different coordinate systems, the vectors and matrices have a left superscript " $k$ " when defined in $\left\{{ }^{k} \boldsymbol{x}_{i}\right\}$, while they have no such superscripts in $\left\{\boldsymbol{x}_{\mathrm{i}}\right\}$.

In the coordinate system $\left\{{ }^{\mathrm{k}} \boldsymbol{x}_{\mathrm{i}}\right\}$, Eq. (6) becomes

$$
{ }^{k} \boldsymbol{Y}={ }^{k} \boldsymbol{\alpha}\left({ }^{k} \boldsymbol{\alpha}\right)^{T}
$$

The tensors ${ }^{k} \boldsymbol{\alpha}$ can be calculated from $\boldsymbol{\alpha}$ by

$$
{ }^{k} \boldsymbol{\alpha}=\left({ }^{k} \mathbf{A}\right) \boldsymbol{\alpha}\left({ }^{k} \mathbf{A}\right)^{T}
$$

where the components of ${ }^{k} \mathbf{A}$ is defined as 


$$
a_{\mathrm{ij}}=\left({ }^{k} \boldsymbol{x}_{\mathrm{i}}\right)^{T} \boldsymbol{x}_{\mathrm{j}}
$$

The transformation of a second-rank tensor can be found in Chapter I in ref. [23]. Because $\boldsymbol{b}_{k}$ is set to lie along ${ }^{k} \boldsymbol{x}_{3}$, only the last element of the matrix $\boldsymbol{b}_{k} \boldsymbol{b}_{k}{ }^{\mathrm{T}}$ is nonzero. Therefore, according to Eq. (6), the last element of ${ }^{k} \boldsymbol{Y}$ (at column 3 and row 3),

$$
{ }^{k} \gamma=\left({ }^{k} \alpha_{31}\right)^{2}+\left({ }^{k} \alpha_{32}\right)^{2}+\left({ }^{k} \alpha_{33}\right)^{2}
$$

is chosen to reveal dislocations, which is called $\gamma$-parameter in this study. Substituting Eq. (8) into Eq. (7), it is not difficult to obtain that

$$
{ }^{k} \gamma=\sum_{j=1}^{3}\left(a_{31} \alpha_{1 \mathrm{j}}+a_{32} \alpha_{2 \mathrm{j}}+a_{33} \alpha_{3 \mathrm{j}}\right)^{2}
$$

From Eq. (11) it is seen that that the $\gamma$-parameter is a function of $a_{31}, a_{32}$ and $a_{33}$, and thus it depends only on ${ }^{k} \boldsymbol{x}_{3}$, which is selected to be parallel to $\boldsymbol{b}_{k}$, but not on ${ }^{k} \boldsymbol{x}_{1}$ and ${ }^{k} \boldsymbol{x}_{2}$. Therefore, ${ }^{k} \boldsymbol{x}_{1}$ and ${ }^{k} \boldsymbol{x}_{2}$ can be freely selected in calculation of the $\gamma$-parameter. Both Eqs. (10) and (11) shows that the $\gamma$-parameter is always nonnegative.

The $\gamma$-parameter can be utilized to determine the Burger vector simply by coordinate transformation. Without loss of generality, let us choose $\boldsymbol{b}=\boldsymbol{b}_{\mathrm{m}}$ to provide an example. When $\boldsymbol{b}=\boldsymbol{b}_{\mathrm{m}}$, by use of Eqs. (6) and (7), one can get

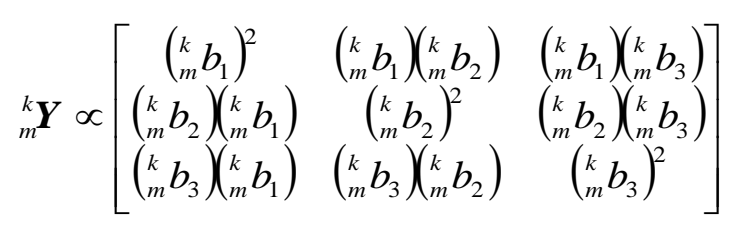

Here the left subscript " $m$ " means that ${ }^{k} \boldsymbol{Y}$ occurs near a dislocation $\boldsymbol{b}_{\mathrm{m}} . \boldsymbol{b}_{m}$ is denoted by ${ }^{k} \boldsymbol{b}_{m}$ in $\left\{{ }^{\mathrm{k}} \boldsymbol{x}_{\mathrm{i}}\right\}$. Assume that ${ }^{k} \boldsymbol{b}_{m}=\left[\begin{array}{lll}{ }_{m}^{k} b_{1} & { }_{m}^{k} b_{2} & { }_{m}^{k} b_{3}\end{array}\right]^{T}$, where ${ }_{m}^{k} b_{1},{ }_{m}^{k} b_{2}$ and ${ }_{m}^{k} b_{3}$ are real numbers. The length of ${ }^{k} \boldsymbol{b}_{m}$ is calculated as $d_{m}=\sqrt{\left({ }_{m}^{k} b_{1}\right)^{2}+\left({ }_{m}^{k} b_{2}\right)^{2}+\left({ }_{m}^{k} b_{3}\right)^{2}}$. Correspondingly, the $\gamma$-parameter in Eq. (10) can be written as

$$
{ }_{m}^{k} \gamma=\left({ }_{m}^{k} \alpha_{31}\right)^{2}+\left({ }_{m}^{k} \alpha_{32}\right)^{2}+\left({ }_{m}^{k} \alpha_{33}\right)^{2}
$$

It is apparent that both $k$ and $m$ take on the values 1 to $n$ in Eqs. (10)- (13). 
When $k=m$, considering that ${ }^{m} \boldsymbol{b}_{m}=\left[\begin{array}{lll}0 & 0 & d_{m}\end{array}\right]^{T}$, Eqs. (12) and (13) changes into Eqs. (14) and (15), respectively, as listed below.

$$
\begin{aligned}
{ }_{m}^{m} \boldsymbol{Y} & \propto\left[\begin{array}{ccc}
0 & 0 & 0 \\
0 & 0 & 0 \\
0 & 0 & d_{m}^{2}
\end{array}\right] \\
{ }_{m}^{m} \gamma={ }_{m}^{m} \gamma_{e}+{ }_{m}^{m} \gamma_{s} & =\left({ }_{m}^{m} \alpha_{31}\right)^{2}+\left({ }_{m}^{m} \alpha_{32}\right)^{2}+\left({ }_{m}^{m} \alpha_{33}\right)^{2}
\end{aligned}
$$

where ${ }_{m}^{m} \gamma_{e}=\left({ }_{m}^{m} \alpha_{31}\right)^{2}+\left({ }_{m}^{m} \alpha_{32}\right)^{2}$ and ${ }_{m}^{m} \gamma_{s}=\left({ }_{m}^{m} \alpha_{33}\right)^{2} \cdot{ }_{m}^{m} \gamma_{e}$ and ${ }_{m}^{m} \gamma_{s}$ arise from edge and screw components, respectively, of a dislocation $\boldsymbol{b}_{\mathrm{m}}$.

Comparing Eqs. (12) with (14), it is found that ${ }_{m}^{k} \gamma$ reaches its maximum value ${ }_{m}^{m} \gamma$ because the corresponding variable $\left({ }_{m}^{k} b_{3}\right)^{2}$ will never increase beyond $d_{m}^{2}$. In other words, the $\gamma$-parameter near the line of a dislocation $\boldsymbol{b}_{m}$ will become largest when the coordinate system is selected to be $\left\{{ }^{m} \boldsymbol{x}_{i}\right\}$, the one specially established for $\boldsymbol{b}_{m}$. This could be used to determine the Burgers vectors of dislocations. In addition, ${ }_{m}^{m} \gamma$ is positive at dislocation cores and zero in other regions. This can be used to discriminate atoms in dislocation cores from others.

\section{Applications}

Atomistic simulations are performed to testify the forgoing deduction of the $\gamma$-parameter and the capabilities of an approach developed on the basis of the $\gamma$-parameter. Several bcc iron single crystals with different size and a face centered cubic copper single crystal are created, respectively, in the simulation box with three axes $\boldsymbol{x}_{1}, \boldsymbol{x}_{2}$ and $\boldsymbol{x}_{3}$. In iron, $\boldsymbol{x}_{1}\left\|[111], \boldsymbol{x}_{2}\right\|[\overline{1} \overline{1} 2]$ and

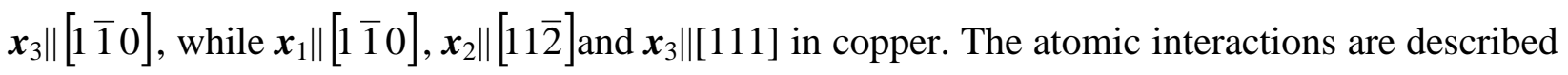
by the embedded-atom-method potentials of iron [24] and copper [25]. The length unit is the lattice parameter $a$, and the $\gamma$-parameter unit is $0.01 a^{-2}$. Periodic boundary conditions are applied along $\boldsymbol{x}_{1}$ and $\boldsymbol{x}_{2}$. Atoms near the two free surfaces perpendicular to $\boldsymbol{x}_{3}$ are fixed, while atoms near the top surface are free in nanoindentation simulation.

Eq. (14) shows that the matrix ${ }_{m}^{m} Y$ contains only one nonnegative element, the $\gamma$-parameter. This deduction is verified first due to its importance for the application of the $\gamma$-parameter. For this purpose, a straight edge dislocation along $\boldsymbol{x}_{2}$ is created in an iron single crystal and fully 
relaxed by molecular statics. Its Burgers vector $[111] / 2$ is $\boldsymbol{b}_{1}=[\sqrt{3} / 200]$ in $\left\{\boldsymbol{x}_{i}\right\}$. The $\gamma$ parameter and the $\boldsymbol{Y}$-matrix are calculated in $\left\{{ }^{1} \boldsymbol{x}_{i}\right\}$, denoted by ${ }^{1} \boldsymbol{Y}$ and ${ }^{1} \gamma$, respectively, in this example. Here $\left\{{ }^{1} \boldsymbol{x}_{i}\right\}$, different from the coordinate system of the simulation box $\left\{\boldsymbol{x}_{i}\right\}$, represents the coordinate system established for the Burgers vector $\boldsymbol{b}_{1}$. Its axis ${ }^{1} \boldsymbol{x}_{3}$ is parallel to the direction [111], but ${ }^{1} \boldsymbol{x}_{1}$ and ${ }^{1} \boldsymbol{x}_{2}$ can be freely selected provided that they can be grouped into the coordinate

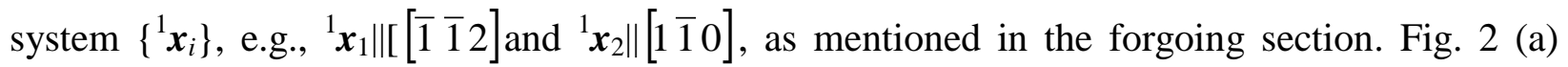
shows that the distribution of ${ }^{1} \gamma$ perpendicular to the dislocation line. As expected, ${ }^{1} \gamma$ is positive at the dislocation core but decays abruptly to zero away from the core. It is obvious that ${ }^{1} \gamma$ can locate the core position precisely. The distribution of all the elements in ${ }^{1} \boldsymbol{Y}$ along the line $p p^{\prime}$ in Fig. 2 (a) is plotted in Fig. 2 (b), from which it is seen that all the elements are almost zero at the dislocation core except ${ }^{1} \gamma$, consistent with Eq. (14). Here note that ${ }^{1} \boldsymbol{Y}$ and ${ }^{1} \gamma$ change into ${ }_{1}^{1} \boldsymbol{Y}$ and ${ }_{1}^{1} \gamma$ in the core region of the dislocation $\boldsymbol{b}_{1}$, that is, $k=m=1$ in Eqs. (13)-(15).

As mentioned above in comparison between Eqs. (12) and (14), ${ }_{m}^{k} \gamma$ reaches its maximum value ${ }_{m}^{m} \gamma$ when $k=m$. This can be clearly seen from the $\gamma$-parameter distribution in a copper single crystal with two partial dislocations $[2 \overline{1} \overline{1}] / 6\left(\boldsymbol{b}_{1}\right)$ and $[\overline{1} 2 \overline{1}] / 6\left(\boldsymbol{b}_{2}\right)$ in Fig. 3 . The $\gamma$ parameters ${ }^{1} \gamma$ in $\left\{{ }^{1} \boldsymbol{x}_{i}\right\}$ and ${ }^{2} \gamma$ in $\left\{{ }^{2} \boldsymbol{x}_{i}\right\}$ are presented in Fig. 3 (a) and (b). It is seen from the spatial distribution of these $\gamma$-parameters that ${ }^{1} \gamma$ and ${ }^{2} \gamma$ can detect core atoms of the dislocations $\boldsymbol{b}_{1}$ and $\boldsymbol{b}_{2}$, respectively. Fig. 3 (c) presents the distribution of ${ }^{1} \gamma$ and ${ }^{2} \gamma$ along $t t^{\prime}$ in Fig. 3 (a) and (b) for further comparison. It is apparent that ${ }_{1}^{1} \gamma\left({ }_{2}^{2} \gamma\right)$ is much larger than ${ }_{1}^{2} \gamma\left({ }_{2}^{1} \gamma\right)$ in the core region of the dislocation $\boldsymbol{b}_{1}\left(\boldsymbol{b}_{2}\right)$ in Fig. 3 (c), consistent with the conclusion that the $\gamma$-parameter in the core region of a dislocation $\boldsymbol{b}_{m}$ is larger than others when expressed in $\left\{{ }^{k} \boldsymbol{x}_{i}\right\}$, where $k=m$.

When the $\gamma$-parameter is extended to more complicated situations, a fully automated approach is needed to determine core atoms and the Burgers vectors of dislocations simultaneously. Its main procedure consists of five steps when implemented into atomistic modeling:

(1) Select the coordinate systems $\left\{\boldsymbol{x}_{\mathrm{i}}\right\}$ and all possible Burgers vectors $\boldsymbol{b}_{k}(k=1 \cdots n)$, and set ${ }^{k} \boldsymbol{x}_{3}=\boldsymbol{b}_{k} /\left|\boldsymbol{b}_{k}\right|$. 
(2) Choose a target atom, at which calculate $\boldsymbol{\alpha}$ in $\left\{\boldsymbol{x}_{i}\right\}$ in the same way as proposed by Hartley and Mishin [14], and then calculate ${ }^{k} \gamma$ from Eq. (11) for $k=1 \cdots n$.

(3) Find the largest one from the calculated $n$ values of ${ }^{k} \gamma(k=1 \cdots n)$, which is assumed to be ${ }^{m} \gamma(k=m)$.

(4) If ${ }^{m} \gamma>\gamma_{c}$, the target atom belongs to a dislocation $\boldsymbol{b}_{m}$ and colored by its Burgers vector, or it will be filtered out.

(5) Repeat steps (2) to (4) over all the simulated atoms.

In step (4), $\gamma_{c}$ is a specified threshold to distinguish atoms in dislocation cores from those out of core regions. It can be obtained when revealing a straight dislocation. For example, we set it to be 1.5 according to Fig. 2 (b) and Fig. 3 (c), and in this case the dislocation core radius is about twice the length of the shortest translation vector. The selected value of $\gamma_{c}$ has a certain effect on the number of core atoms finally detected.

The above approach is employed to visualize and characterize three self-interstitial-atom clusters from a molecular statics simulation, which are created on different $\{111\}$ habit planes. Fig. 4 shows that these interstitial clusters exhibit their feature as a line defect, that is, they are actually small dislocation loops with the Burgers vectors perpendicular to the habit plane of the corresponding interstitial clusters. It is also seen from Fig. 4 that the six-fold symmetrical shape of these loops controlled by the crystal structure are also clearly revealed by our approach. This confirms that this approach is capable of revealing curved dislocations in an accurate fashion. The smallest loop in Fig. 4 contains fourteen interstitials: twelve interstitials are located on the six lattice sites along the loop, and the remaining two on the only lattice site at the loop center. Obviously, it is the smallest possible loop in the simulated iron since it cannot shrink any more as a line defect. Therefore, our approach possesses a high spatial resolution to reveal all possible dislocation loops.

Finally, the approach is implemented into a molecular dynamics simulation of nanoindentation to demonstrate its capability of tracing the evolution of complex dislocation networks on the fly. Fig. 5 shows a complex dislocation network during nanoindentation, simultaneously revealed by a common filtering tool and the $\gamma$-parameter approach for comparison. Because the excess 
energy analysis is not specific to dislocations, as previously mentioned, many additional atoms are involved in Fig. 5 (a) although they are not located in dislocation cores. In contrast, only dislocation core atoms are revealed by our approach in Fig. 5 (b) because the $\gamma$-parameter is devised specifically for dislocations. Furthermore, because the Burgers vectors are revealed in Fig. 5 (b), nucleation of small segments, dissociation and reaction of dislocations and formation of nodes can be characterized. Fig. 5 (c) provides a close-up view of a junction dislocation [100] with two triple nodes, from which it is seen that our approach works well for multiple dislocations that are close to each other. It is worth mentioning that utilizing a filtering tool such as excess energy analysis to discard some atoms far from dislocations can dramatically improve the efficiency of the present approach, but not required.

\section{Discussions}

It is seen clearly from the above examples that our approach successfully extends the application domain of the Nye tensor in atomistic modeling [14] to the curved dislocations and their dynamic evolution under the same high spatial resolution. This approach reduces the nine elements of the Nye tensor to a single scalar $\gamma$-parameter, but still can provide information about the screw and edge components of a dislocation, as seen from Eq. (15). Besides, it depends only on the calculation of the Nye tensor, which can be performed regardless of phase composition. Therefore, our approach can be straightforwardly applied to plastic deformation of intermetallic compound and alloys, in which dislocation cores are dissociated in a rather complicated way [2628]. It is worth mentioning that the $\gamma$-parameter is not the only choice to extend the Nye tensor to curved dislocations, the approach can also be well performed if the $\gamma$-parameter is replaced by a scalar, $\boldsymbol{e}^{\mathrm{T}} \boldsymbol{Y} \boldsymbol{e}$, where $\boldsymbol{e}=\boldsymbol{b} /|\boldsymbol{b}|$ and $\boldsymbol{Y}$ is defined in Eq. (6). This scalar will be largest regardless of the selection of coordinate systems provided that the Burgers vector is properly selected.

The automated approach in the forgoing section cannot distinguish two types of dislocations with parallel Burgers vectors with different length. However, it is seen from Eq. (14) that the $\gamma$ parameter of a dislocation $\boldsymbol{b}_{\mathrm{m}}$ in $\left\{{ }^{m} \boldsymbol{x}_{i}\right\}$ is proportional to the square of the length of the Burgers vector $\boldsymbol{b}_{\mathrm{m}}$. Therefore, the $\gamma$-parameter should be different for parallel Burger vectors with different length. This could be useful for further classification of dislocations with parallel Burgers vectors. In addition, this work is restricted on dislocations in a single crystal, but it can be extended to polycrystalline materials with boundaries $[29,30]$ provided the lattice distortion 
tensor $\mathbf{G}$ can be properly calculated [14]. These applications will be conducted in our future work.

\section{Conclusions}

In summary, a $\gamma$-parameter has been proposed from the Nye tensor. The two main features of this scalar parameter are that it is positive at dislocation cores and zero otherwise, and that its maximum peak value occurs at a dislocation, which is independent on the change of the dislocation line direction, in the coordinate system specially established according to the Burgers vector. On the basis of the $\gamma$-parameter, a fully automated approach has been developed to locate dislocation cores and determine their Burgers vectors at the same time. It has the same high resolution as the Nye tensor method, and can be readily implemented into atomistic modeling to visualize the evolution of complex dislocation networks on the fly.

\section{Acknowledgements}

This research was supported by the Program of "One Hundred Talented People" of the Chinese Academy of Sciences (JBY), the National Natural Science Foundation of China (NSFC) under grant No. 51331007, the U.S. Department of Energy, Office of Basic Energy Sciences, Materials Sciences and Engineering Division (YNO and JBY) and the Office of Fusion Energy Sciences (RES). 


\section{References}

[1] D. Wolf, V. Yamakov; S.R. Phillpot, Acta Mater. 53 (2005) 1.

[2] M.A. Tschopp, D.L. McDowell, J. Mech. Phys. Solids 56 (2008) 1806.

[3] M.A. Tschopp, D.L. McDowell, Scripta Mater. 58 (2008) 299.

[4] C. Begau, A. Hartmaier, E.P.George, G.M. Pharr, Acta Mater. 59 (2011) 934.

[5] I. Salehinia, S.K. Lawrence, D.F. Bahr, Acta Mater. 61 (2013) 1421.

[6] V.V. Bulatov, W. Cai, Computer Simulation of dislocations. Oxford: Oxford University Press; 2006.

[7] J.D. Honeycutt, H.C. Anderren, J. Phys. Chem. 91 (1987) 4950.

[8] A. Stukowski, Modelling Simul. Mater. Sci. Eng. 20 (2012) 045021.

[9] A. Stukowski, K. Albe, Modelling Simul. Mater. Sci. Eng. 18 (2010) 025016.

[10] A. Stukowski, K. Albe, Modelling Simul. Mater. Sci. Eng. 18 (2010) 085001.

[11] J.B. Yang, Y. Nagai, M. Hasegawa, Y.N. Osetsky, Philos. Mag. 90 (2010) 991.

[12] S.C. Wang, G. Lu, G.G. Zhang, Compu. Mater. Sci., 68(2013)396.

[13] A. Stukowski, J. Mech. Phys. Solids. 70 (2014) 314.

[14] C.S. Hartley, Y. Mishin, Acta Mater. 53 (2005) 1313.

[15] B.G. Mendis, Y. Mishin, C.S. Hartley, K.J. Hemker, Philos. Mag. 86 (2006) 4607.

[16] C. Woodward, D.R. Trinkle, L.G. Hector Jr., D.L. Olmsted, Phys. Rev. Lett. 100 (2008) 045507.

[17] C. Begau, J. Hua, A. Hartmaier, J. Mech. Phy. Solids, 60 (2012) 711.

[18] L.L. Li, P. Zhang, Z.J. Zhang, H.F. Zhou, S.X. Qu, J.B. Yang, Z.F. Zhang, Acta Mater. 73 (2014) 167.

[19] J.F. Nye, Acta Metall. 1 (1953) 153.

[20] B. A. Bilby, R. Bullough, E. Smith, Proc. Roy. Soc. A, 231 (1955) 263.

[21] E. Kröner, International J. Solids and Struct. 38 (2001) 1115.

[22] J. Kioseoglou, G.P. Dimitrakopulos, P. Komninou, T. Karakostas, E.C. Aifantis, J. Phys. D 41 (2008) 035408.

[23] J.F. Nye, Physical Properties of Crystals. Oxford: Clarendon Press; 1957. 
[24] G.J. Ackland, M.I. Mendelev, D.J. Srolovitz, S. Han, A.V. Barashev, J. Phys. 16 (2004) S2629.

[25] G.J.Ackland, D.J.Bacon, A.F.Calder, T.Harry, Philos. Mag. A 75 (1997)713.

[26] J.K. Ternes, D. Farkas, R. Kriz, Philos. Mag. A 72 (1995) 1671.

[27] C. Vailhe, D. Farkas, Acta Mater. 45 (1997) 4463.

[28] Y.S. Lin, M. Cak, V. Paidar, V. Vitek, Acta Mater. 60 (2012) 881.

[29] A. Stukowski, V. V. Bulatov, A. Arsenlis, Modelling Simul. Mater. Sci. Eng. 20 (2012), 085007 .

[30] M. Elsey, B. Wirth, Multiscale Modeling \& Simul. 12 (2014) 1. 


\section{Figure captions:}

Fig. 1. Two types of coordinate systems for dislocation visualization. One is the coordinate system of the simulation box with three axes $\boldsymbol{x}_{1}, \boldsymbol{x}_{2}$ and $\boldsymbol{x}_{3}$, and the other has three axes ${ }^{k} \boldsymbol{x}_{1},{ }^{k} \boldsymbol{x}_{2}$ and ${ }^{k} \boldsymbol{x}_{3}$. The latter is designed expressly $\left({ }^{k} \boldsymbol{x}_{3} \| \boldsymbol{b}_{k}\right)$ for visualization of all dislocations with a Burgers vector $\boldsymbol{b}_{k}$ in the simulation box. Only one dislocation loop $\boldsymbol{b}_{k}$ is sketched for clarity.

Fig.2. (a) The spatial distribution of ${ }^{1} \gamma$ on the plane $(11 \overline{2})$ for an edge dislocation [111]/2 (b) in iron. (b) The distribution of the nine elements in ${ }^{1} Y$ along $p p^{\prime}$ in (a). ${ }^{1} \gamma$ is denoted by red circles in (b), while all other elements by black squares because all of them are almost zero. The shaded area in (b) is drawn only as visual guides to show the peak of the ${ }^{1} \gamma$ distribution along $p p^{\prime} . a_{\mathrm{fe}}$ is the lattice parameter of iron.

Fig.3. The spatial distribution of (a) ${ }^{1} \gamma$ and (b) ${ }^{2} \gamma$ on the plane $(11 \overline{2})$ for two partial dislocations $[2 \overline{1} \overline{1}] / 6\left(\boldsymbol{b}_{1}\right)$ and $[\overline{1} 2 \overline{1}] / 6\left(\boldsymbol{b}_{2}\right)$, produced by dissociation of an edge dislocation $[1 \overline{1} 0] / 2$ on a plane (111) in a copper single crystal. (c) The distribution of ${ }^{1} \gamma$ and ${ }^{2} \gamma$ along $t t^{\prime}$ in (a) and (b). ${ }^{1} \gamma$ and ${ }^{2} \gamma$ denote the $\gamma$-parameter calculated in $\left\{{ }^{1} \boldsymbol{x}_{\mathrm{i}}\right\}$ and $\left\{{ }^{2} \boldsymbol{x}_{\mathrm{i}}\right\}$, respectively. In (c), ${ }^{1} \gamma$ (solid line) is called as ${ }_{1}^{1} \gamma$ at the core of the partial dislocation $\boldsymbol{b}_{1}$, while as ${ }_{2}^{1} \gamma$ at the core of the partial dislocation $\boldsymbol{b}_{2}$, and additionally, ${ }^{2} \gamma$ (dotted line) is termed as ${ }_{1}^{2} \gamma$ at the core of the partial dislocation $\boldsymbol{b}_{1}$, but as ${ }_{2}^{2} \gamma$ at the core of the partial dislocation $\boldsymbol{b}_{2} . a_{\mathrm{cu}}$ is the lattice parameter of copper.

Fig. 4. Three different dislocation loops $<111>/ 2$ in a simulation box with a size of $44.2 \times 58.8 \times 58.0 a^{3}$. Their projection along their Burgers vectors are also presented on the right side for clarity. The dislocation loops $[111] / 2$ (red), $[11 \overline{1}] / 2$ (green) and $[\overline{1} 11] / 2$ (blue) are 
composed of 14, 38 and 122 interstitial iron atoms, respectively.

Fig. 5. An intermediate state of complicated dislocation networks revealed by (a) excess energy analysis and (b) the $\gamma$-parameter, respectively, in association with nanoindentation of an iron single crystal with a size of $191.4 \times 191.1 \times 149.9 a^{3}$ at $300 \mathrm{~K}$. (c) A close-up view of a junction dislocation with two nodes in (b). Dislocation cores are colored according to the list: $\pm[11 \overline{1}] / 2$ red, $\pm[1 \overline{1} 1] / 2$-magenta, $\pm[\overline{1} 11] / 2$-orange, $\pm[111] / 2$-green, $\pm[100]$-cyan, $\pm[010]$-yellow and $\pm[001]$-blue. The arrow indicates a dislocation dissociation: $[001] \rightarrow[\overline{1} \overline{1} 1] / 2+[111] / 2$. 


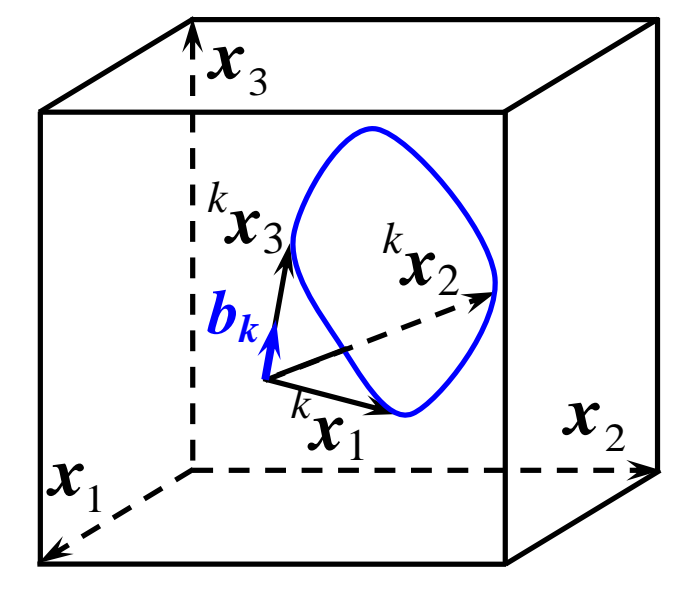

Fig. 1.

.

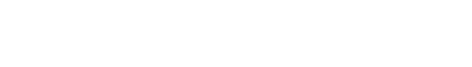

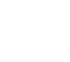

.

(

.

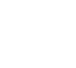

.

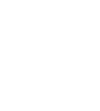
(

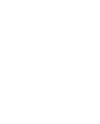

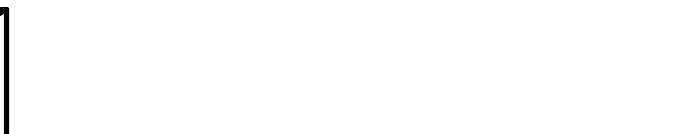

(n) 
(a)

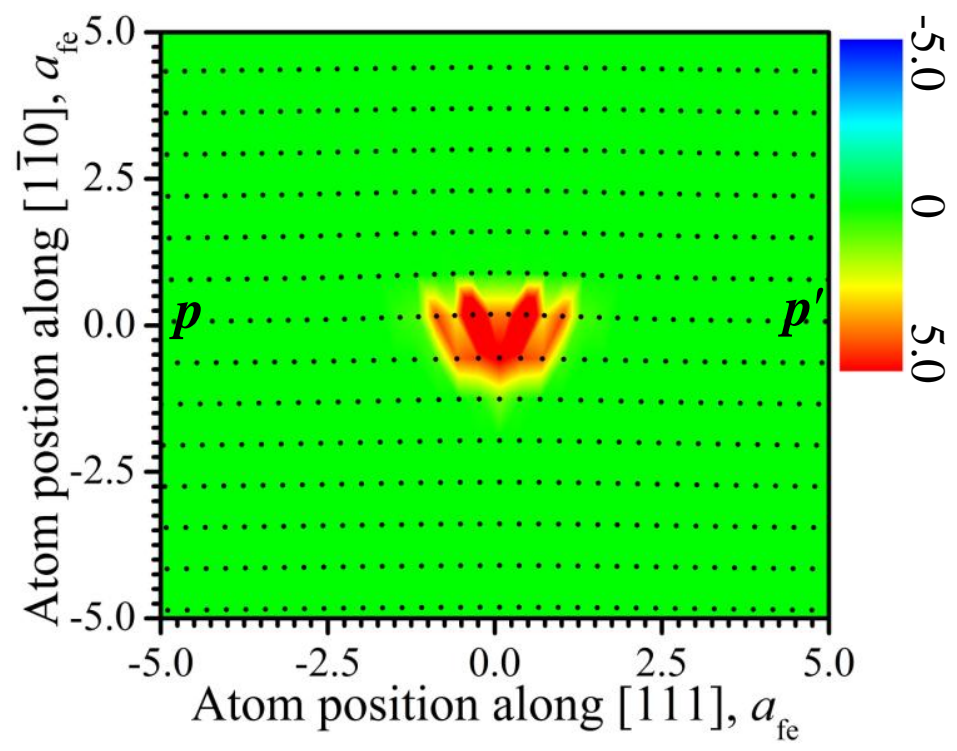

(b)

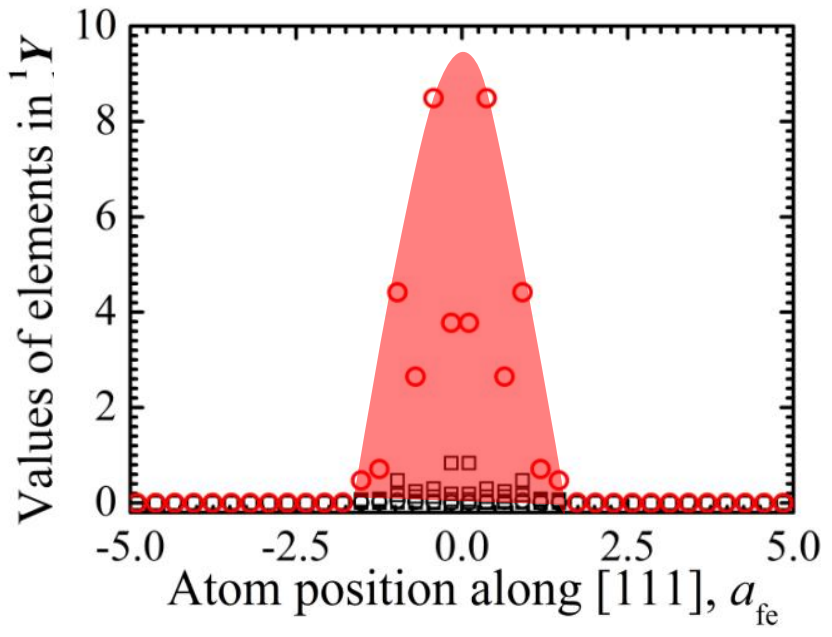

Fig. 2. 
(a)

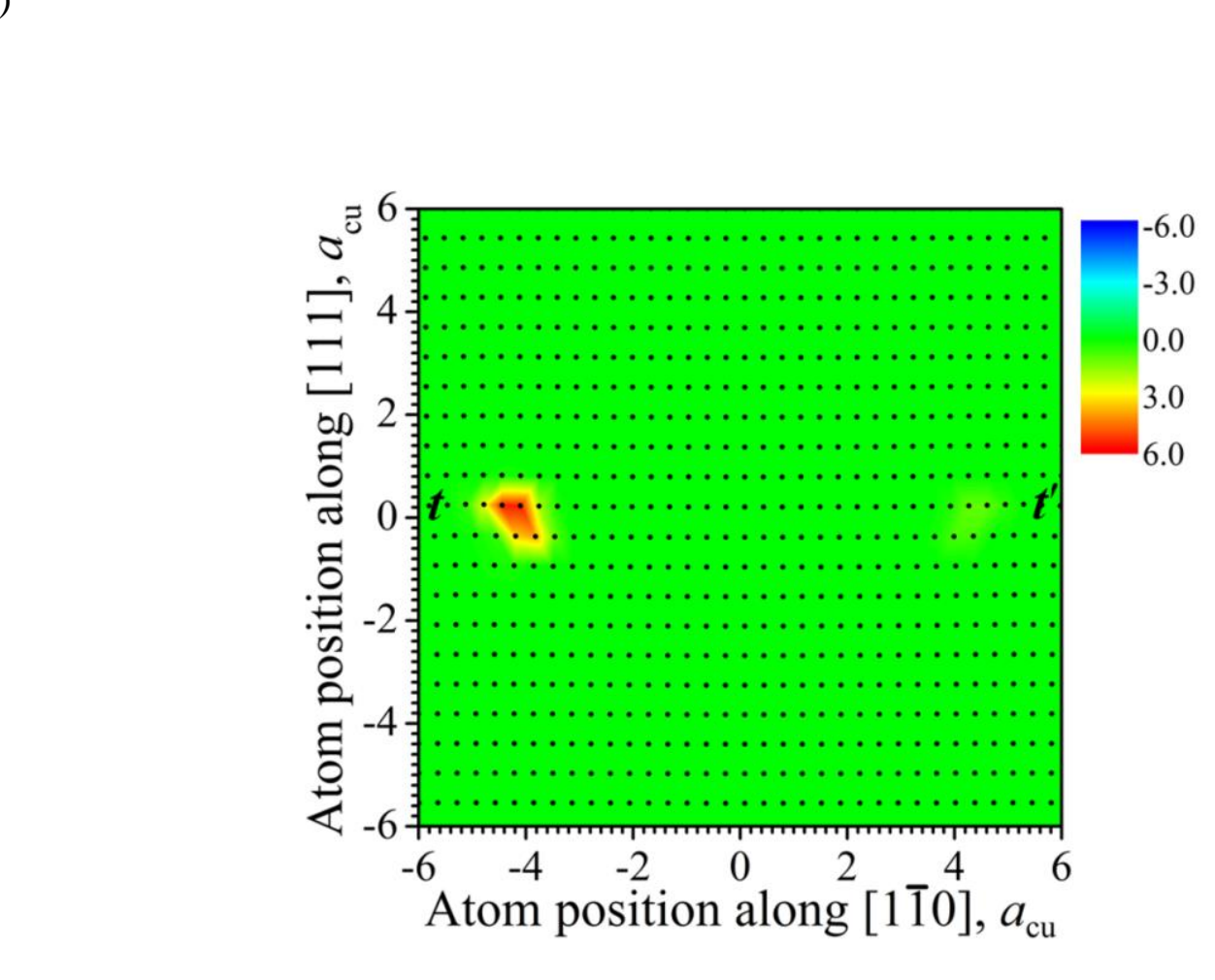

Fig. 3

Figure $-3.0$

.

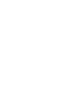

\section{Figure}

(a)

.

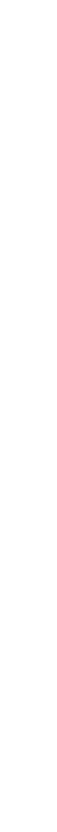


(b)

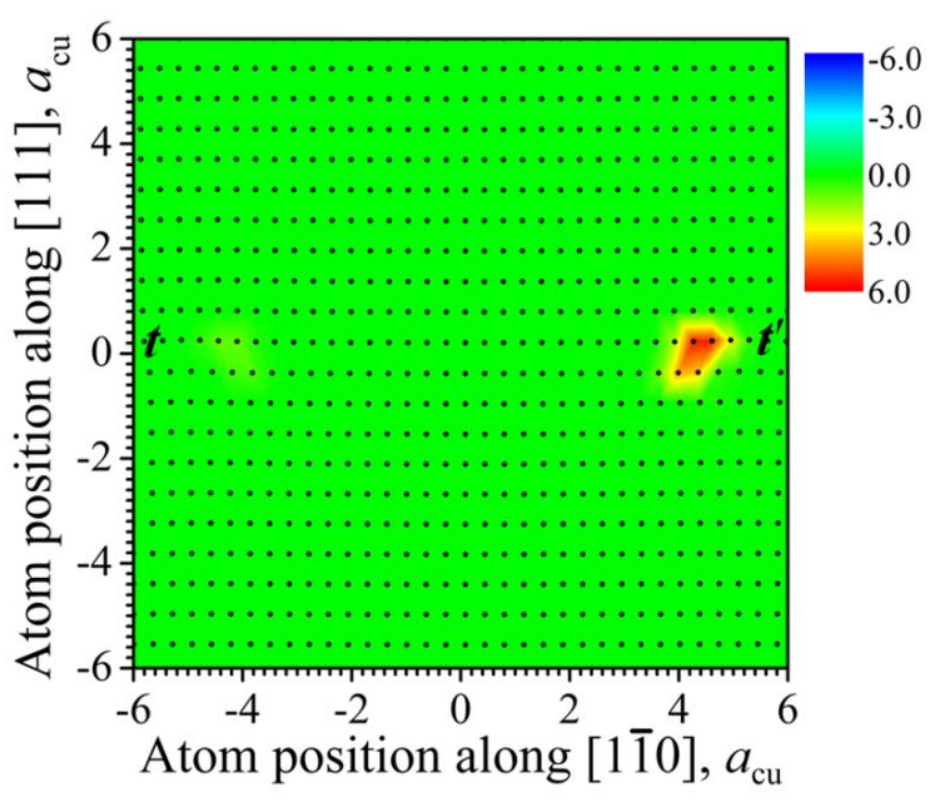

Figure

.0
0
0
0


(c)

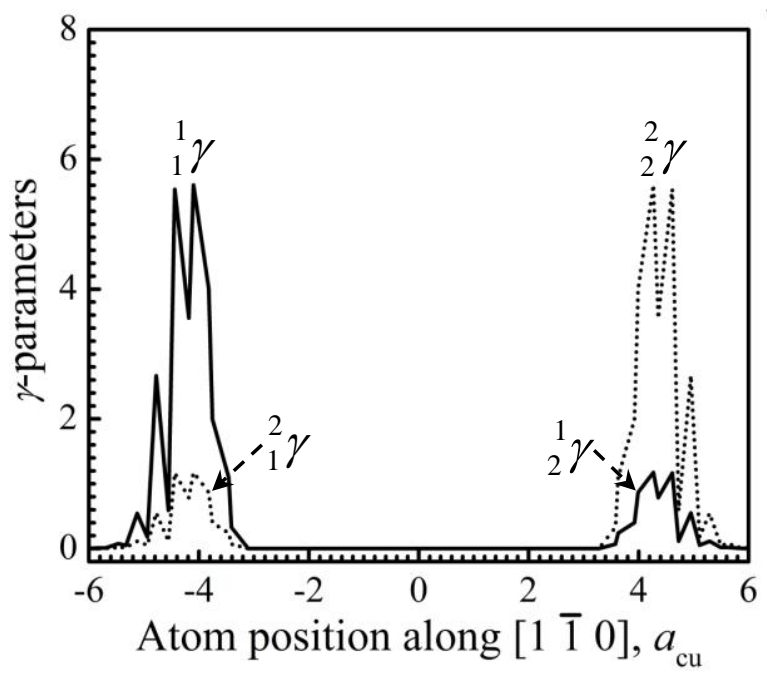

Fig. 3 
Figure

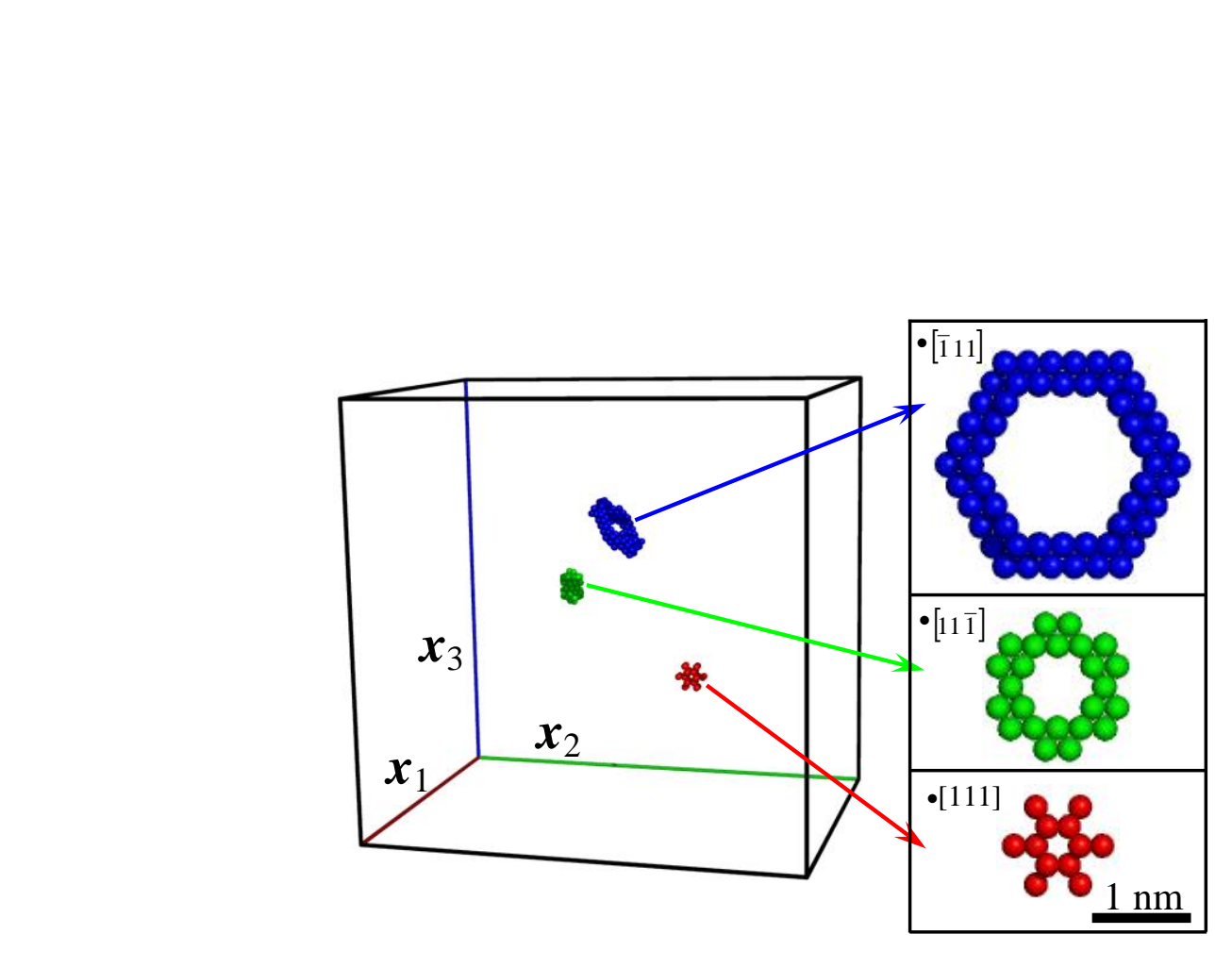

Fig. 4.

\section{Figure}$$
\text { . }
$$

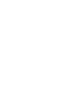$$
\text { . }
$$
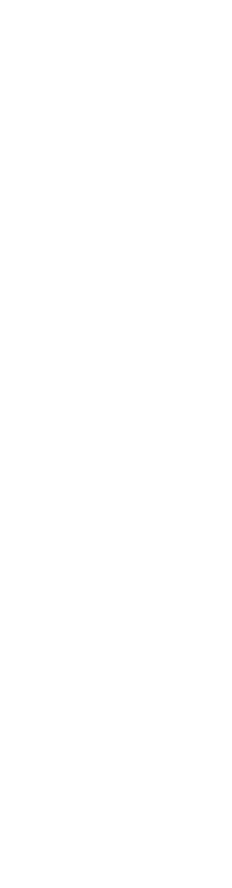
(a)

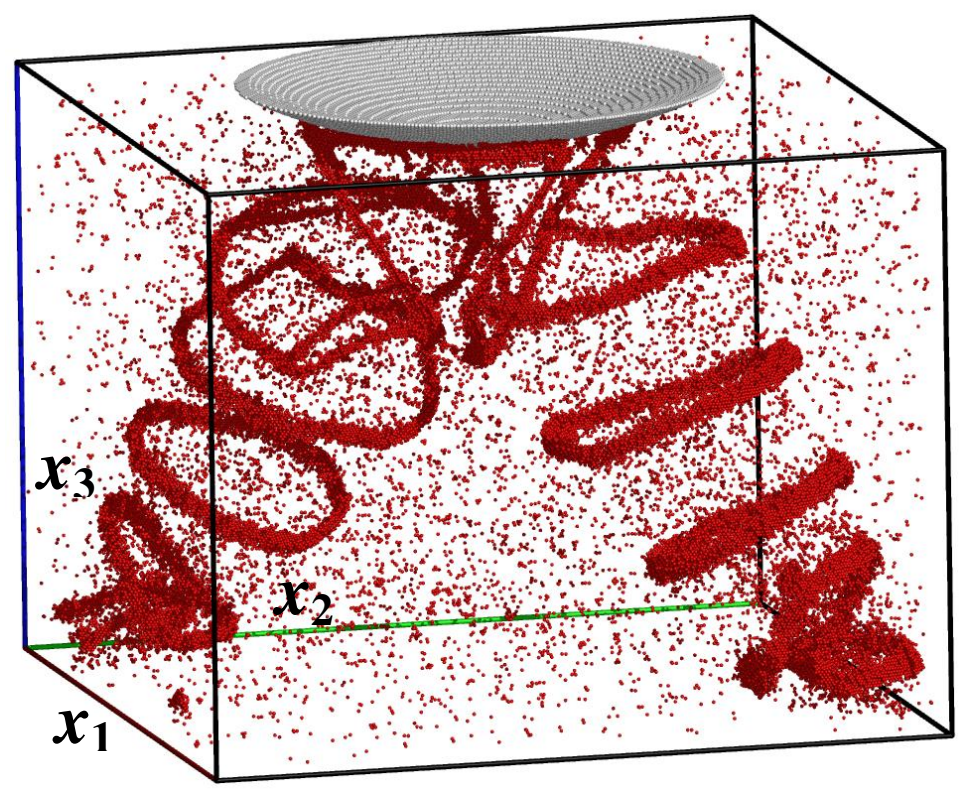

(b)

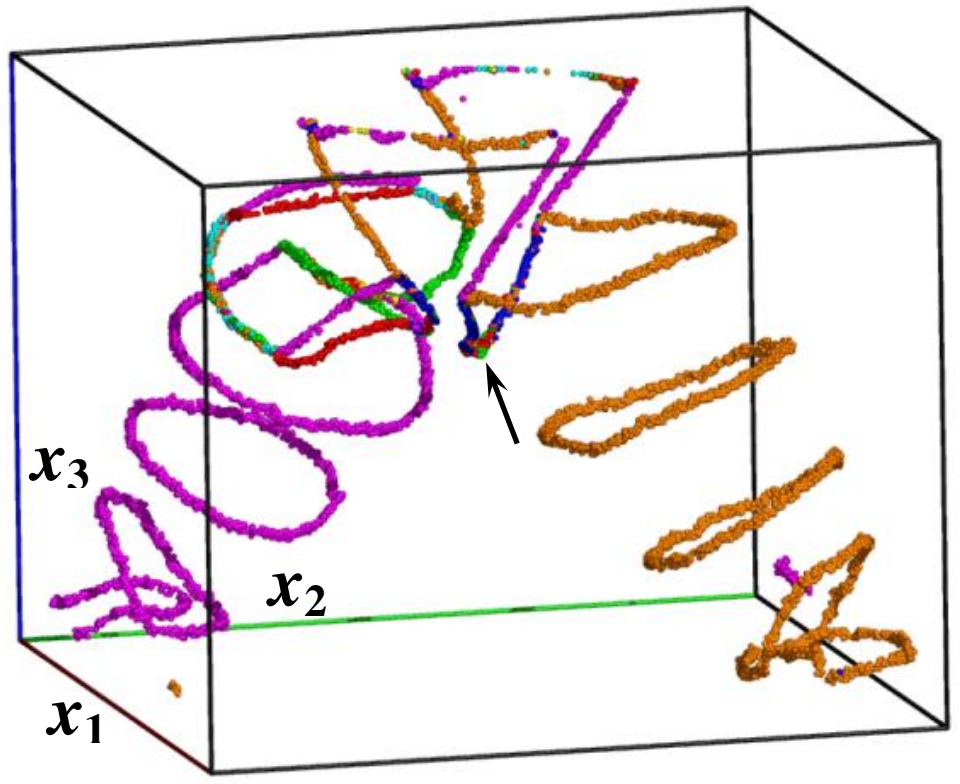


(c)

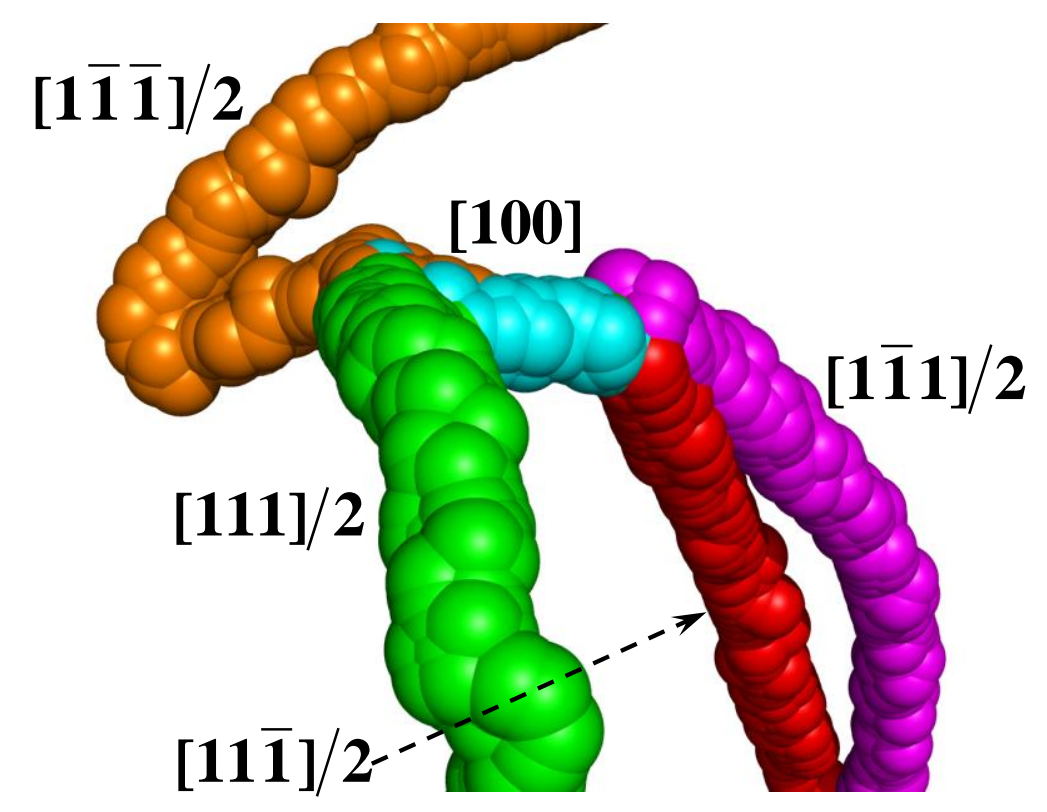

Fig. 5. 
Graphical Abstracts (for review)

Characterization and visualization of tangled curved dislocations on the basis of the Nye tensor calculation only
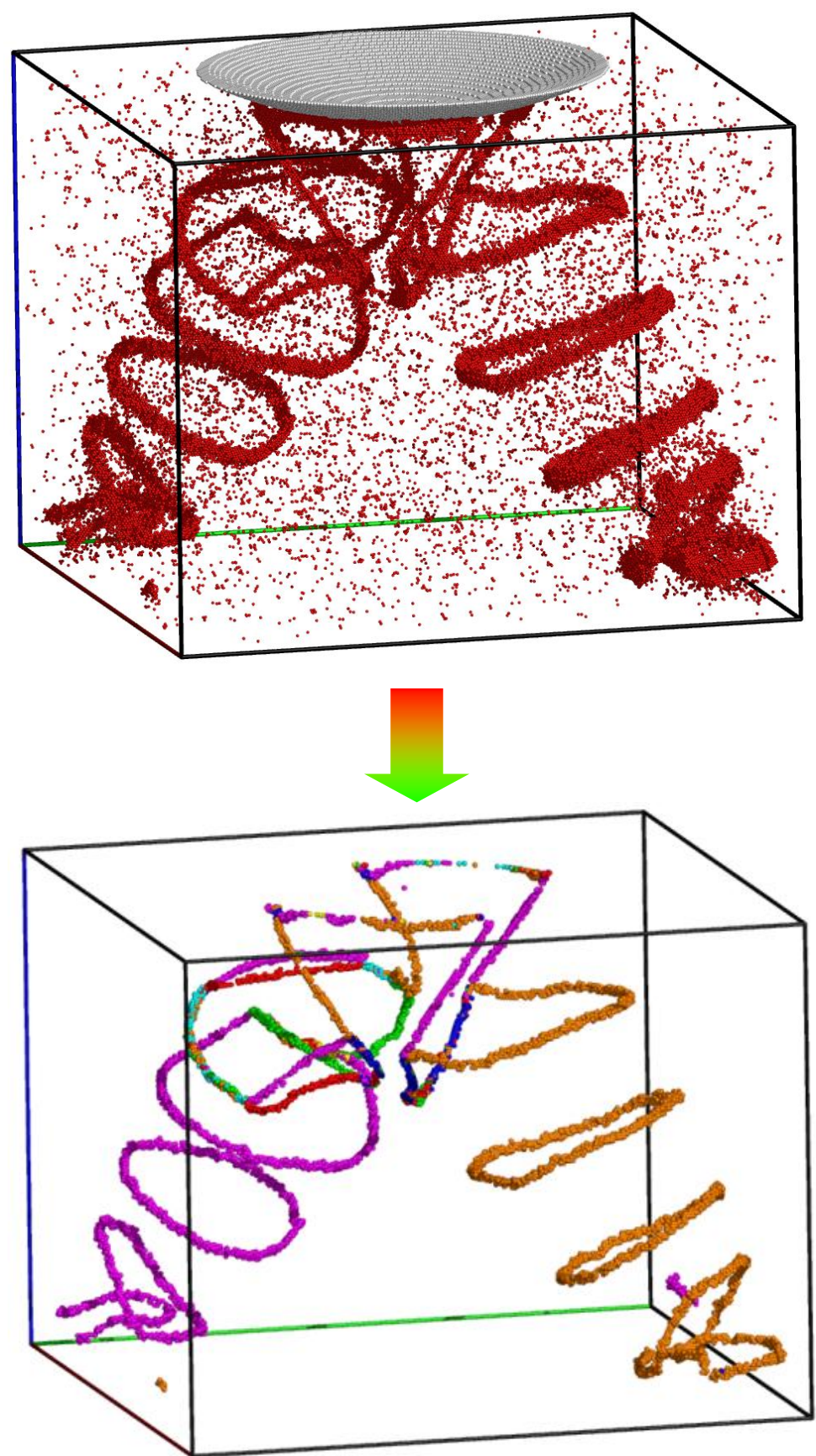